\author{
Motohiro Akagi • Koji Inui • Shigeo Nakajima \\ Masaaki Shima • Toshinori Nishigaki \\ Takashi Muramatsu • Chikara Kokubu \\ Hiroko Tsukamoto $\cdot$ Norio Sakai $\cdot$ Shintaro Okada
}

\title{
Mutation analysis of two Japanese patients with Fanconi-Bickel syndrome
}

\begin{abstract}
Fanconi-Bickel syndrome (FBS), or glycogen storage disease type $\mathrm{XI}$, is a rare autosomal recessive disorder characterized by hepatorenal glycogen accumulation, Fanconi nephropathy, and impaired utilization of glucose and galactose. Recently, this disease was elucidated to link mutations in the glucose transporter 2 (GLUT2) gene. Only three mutations in three FBS families have been reported. Therefore, it is important to elucidate mutations in the GLUT2 gene in FBS by answering the question of whether the syndrome is a single gene disease. In this report, we describe two patients in two unrelated families clinically diagnosed with FBS. No mutation in the entire protein coding region of the GLUT2 gene was detected in patient 1, which suggested that no mutation existed in the GLUT 2 gene, or that some mutations had affected the expression of the GLUT 2 gene. In patient 2, a novel homozygous nonsense mutation (W420X, Trp at codon 420 to stop codon) was detected. These results support the correlation between GLTU2 gene mutation and FBS syndrome. However, many patients must be analyzed to determine whether other genes are involved in FBS.
\end{abstract}

Key words Fanconi-Bickel syndrome - Glycogen storage disease type XI - Glucose transporter $2 \cdot$ Nonsense mutation - Japanese patient

\section{Introduction}

Fanconi-Bickel syndrome (FBS), or glycogen storage disease type $\mathrm{XI}$, is a rare autosomal recessive metabolic disorder characterized by hepatorenal glycogen accumulation, Fanconi nephropathy, and impaired metabolism of glucose

M. Akagi $\cdot$ K. Inui $(\bowtie) \cdot S$. Nakajima $\cdot$ M. Shima $\cdot$ T. Nishigaki · T. Muramatsu $\cdot$ C. Kokubu $\cdot$ H. Tsukamoto $\cdot$ N. Sakai $\cdot$ S. Okada Department of Developmental Medicine (Pediatrics, D-5), Osaka University, Graduate School of Medical Science, 2-2 Yamadaoka, Suita, Osaka 565-0871, Japan

Tel. +81-6-6879-3932; Fax +81-6-6879-3939

e-mail: koji@ped.med.osaka-u.ac.jp and galactose. The disease was first described by Fanconi and Bickel in 1949 (Fanconi and Bickel 1949) and is also known by other names, such as glycogen storage disease type XI. However, the underlying enzymatic defects in carbohydrate metabolism have not been identified. In 1997, Santer et al. (Santer et al. 1997) reported mutations in the glucose transporter 2 (GLUT2) gene in this disease.

The structural gene encoding GLUT2 has been cloned and mapped to chromosome 3q26.1-26.3 (Takeda et al. 1993). The GLUT2 gene contains 11 exons spanning $30 \mathrm{~kb}$. To date, three mutations in the GLUT2 gene have been reported in FBS (Santer et al. 1997). In their recent review of FBS, Santer et al. (1997) described 82 cases from 70 families in the published literature and their personal communications, but only 14 were confirmed, including the reported cases of FBS determined by detection of mutations within the GLUT2 gene. Therefore it is important to elucidate molecular defects to determine whether or not the syndrome is a single gene disease. In this report, we describe two patients with FBS; one had a novel nonsense mutation, but no mutation was detected in the other. This is the first report of the GLUT2 gene mutation in a Japanese patient with FBS.

\section{Case reports}

\section{Patient 1}

The patient was a 23-year-old Japanese woman born by normal delivery of non-consanguineous parents. At 5 months of age, her mother noticed marked distention of her abdomen. Hepatomegaly, glucosuria, and short stature were noted by a local doctor. At 10 months of age, she was diagnosed with renal glucosuria and rickets caused by renal tubular dysfunction. At 4 years of age, she was referred to the National Children's Medical Research Center for a liver biopsy. She was diagnosed with glycogen storage disease with Fanconi syndrome because of increased glycogen accumulation, with normal enzymatic activities of hepatic glu- 
cose-6-phosphatase, glycogen-debranching enzyme, and phosphorylase kinase. She also exhibited galactosuria. Treatments were supplementation of active vitamin D, phosphorus, and bicarbonate, and taking uncooked cornstarch before sleep. Her bone abnormalities, especially genu valgum, gradually progressed. She received an operation for bone deformities. Her final height was $135.0 \mathrm{~cm}$ $(-4.3 \mathrm{SD})$ and body weight was $36.4 \mathrm{~kg}(-2.3 \mathrm{SD})$. Her two sisters were healthy.

\section{Patient 2}

The patient was a 21-year-old Japanese man born by normal delivery from consanguineous parents (his grandmothers were cousins). Poor weight gain was detected at 1 month of age, and hepatomegaly and glucosuria were found. He was diagnosed with Fanconi syndrome at 3 months of age. At the age of 1 year and 6 months, he received a liver biopsy for definitive diagnosis, but enzymatic activities of glycogen metabolism were all normal, despite an increased amount of glycogen. He also showed galactosuria and was diagnosed with glycogen storage disease and Fanconi syndrome, and was treated with vitamin $\mathrm{D}$, phosphorus, and bicarbonate. He was also given uncooked cornstarch before sleep. At the age of 20 years, brain computed tomography scans showed calcification of the bilateral basal ganglia, while renal calcification was detected by ultrasonography. His final height was $148.5 \mathrm{~cm}$ $(-3.0 \mathrm{SD})$ and body weight was $37.0 \mathrm{~kg}(-2.8 \mathrm{SD})$. His sister was healthy.

\section{Materials and methods}

Genomic DNA was isolated from peripheral blood leukocytes of the patients and their parents by standard methods. All 11 exons of the GLUT 2 gene, including the exon/intron boundary, were amplified from genomic DNA by polymenase chain reaction (PCR) methods, using the primer sets noted by Takeda et al. (1993). In patient 1, total RNA was isolated from cultured skin fibroblasts and subjected to reverse transcribed-PCR (RT-PCR) amplification. The amplified products were purified and directly sequenced with a dye terminator cycle sequencing ready reaction kit (ABI 310 Genetic Analyzer, Perkin-Elmer, Foster City, CA, USA).

\section{Results and discussion}

FBS is characterized by hepatorenal glycogen storage, hypoglycemia, hypergalactosemia, and proximal renal tubular dysfunction. Recently GLUT2 gene mutations were reported in FBS (Santer et al. 1997). The two patients reported here had all the characteristics of and were clinically diagnosed with FBS. However, DNA sequence analysis revealed that patient 2 had a G-to-A transition at cDNA nucleotide number 1159 in exon 9 of the GLUT 2 gene, resulting in a Trp to stop codon at codon 420 (TGG to TAG, designated as W420X) (Fig. 1). No mutation was detected in the entire protein coding region of the GLUT2
Fig. 1. Direct sequence analysis of genomic DNA of the GLUT2 gene. Direct sequence analysis of the exon 9 region in patient 2 showed a homozygous amino acid of codon 420 (TAG; underlined) in the patient, and a heterozygous amino acid of the codon ( $T G G$ and $T A G$; underlined $)$ in his parents. The peak heights of $\mathrm{G}$ and $\mathrm{A}$ were the same and the analyses were designated $N$
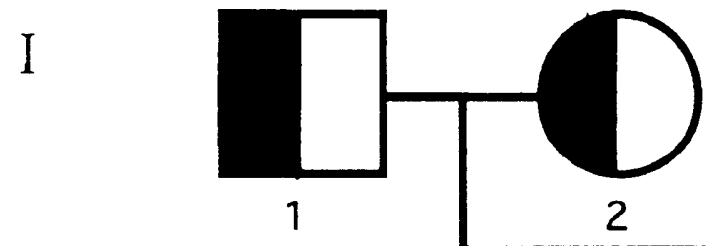



1

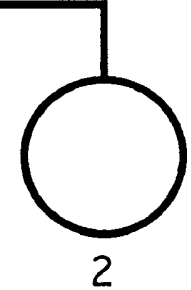

2

gene in patient 1 . The mutation in patient 2 was confirmed to be heterozygous (G/A) in his parents for the W420X mutation (Fig. 1).

To examine mRNA in patient 1 and a control, RT-PCR was performed using cultured skin fibroblasts from the patient and control. However, it was impossible to amplify cDNA of the GLUT 2 gene from normal cultured skin fibroblasts, probably because there was no expression of the gene in the cells (Fukumoto et al. 1988). In patient 1, two possibilities were considered; no mutation existed in the GLUT 2 gene, or, alternatively, some mutations affected the expression of the GLUT 2 gene. Moreover, there was a low possibility that deletion mutations were present in the primer sequence.

To date, four mutations in the GLUT2 gene, including the one in this report, have been reported in FBS; three were nonsense mutations and one was a deletion (Santer et al. 1997). All reported mutations had a stop codon, resulting in truncated protein without functional monosaccharide transport activity. However, there are reports of a missense mutation in the GLUT2 gene in a diabetic patient and amino acid polymorphisms in the normal population (Mueckler et al. 1994; Matsubara et al. 1995; Shimada et al. 1995). In FBS, the reported mutations are very few, and no missense mutation has been reported. The phenotype of a missense mutation of the GLUT 2 gene is speculative as is the correlation of diabetes and GLUT2 gene mutations. Moreover, from the limited evidence it cannot be concluded that FBS is a single gene disease caused by GLUT2 gene mutations. Further mutation analysis could disclose whether FBS is a single gene disease or whether it is a syndrome derived from different gene loci.

\section{References}

Fanconi G, Bickel H (1949) Die chronische Aminoacidurie (Aminosaurediabetes oder nephrotisch-glukosurischer Zwergwuchs) bei der Glykogenose und der Cystinkrankheit. Helv Pediatr Acta 4:359-396

Fukumoto H, Seino S, Imura H, Seino Y, Eddy RL, Fukushima Y, Byers MG, Shows TB, Bell GI (1988) Sequence, tissue distribution, and chromosomal location of mRNA encoding a human glucose transporter-like protein. Proc Natl Acad Sci USA 85:5434-5438

Matsubara A, Tanizawa Y, Matsutani A, Kaneko T, Kaku K (1995) Sequence variations of the pancreatic islet/liver glucose transporter (GLUT2) gene in Japanese subjects with noninsulin-dependent diabetes mellitus. J Clin Endocrinol Metab 80:3131-3135

Mueckler M, Kruse M, Strube M, Riggs AC, Chiu KC, Permutt MA (1994) A mutation in the glut2 glucose transporter gene of a diabetic patient abolishes transport activity. J Biol Chem 269:17765-17767

Santer R, Schneppenheim R, Dombro A, Gotze H, Steinmann B, Schaub J (1997) Mutations in GLUT2, the gene for the liver-type glucose transporter, in patients with Fanconi-Bickel syndrome. Nat Genet 17:324-326

Shimada F, Makino H, Iwaoka $\mathrm{H}$, Miyamoto S, Hashimoto N, Kenatsuka A, Bell GI, Yoshida S (1995) Identification of two novel amino acid polymorphisms in beta-cell/liver (GLUT2) glucose transporter in Japanese subjects. Diabetologia 38:n211-215

Takeda J, Kayano T, Fukomto H, Bell GI (1993) Organization of the human GLUT2 (pancreatic $\beta$-cell and hepatocyte) glucose transporter gene. Diabetes 42:773-777 